MATHEMATICS OF COMPUTATION

Volume 67, Number 221, January 1998, Pages 191-207

S $0025-5718(98) 00898-9$

\title{
COMPACTLY SUPPORTED TIGHT AFFINE SPLINE FRAMES IN $L_{2}\left(\mathbb{R}^{d}\right)$
}

\author{
AMOS RON AND ZUOWEI SHEN
}

\begin{abstract}
The theory of fiberization is applied to yield compactly supported tight affine frames (wavelets) in $L_{2}\left(\mathbb{R}^{d}\right)$ from box splines. The wavelets obtained are smooth piecewise-polynomials on a simple mesh; furthermore, they exhibit a wealth of symmetries, and have a relatively small support. The number of "mother wavelets", however, increases with the increase of the required smoothness.

Two bivariate constructions, of potential practical value, are highlighted. In both, the wavelets are derived from four-direction mesh box splines that are refinable with respect to the dilation matrix $\left(\begin{array}{cc}1 & 1 \\ 1 & -1\end{array}\right)$.
\end{abstract}

\section{INTRODUCTION}

Given a finite set $\Psi \subset L_{2}\left(\mathbb{R}^{d}\right)$, and a dilation matrix $s$, the affine system generated by $\Psi$ is defined as the collection

$$
X:=\left\{D^{k} E^{\alpha} \psi: \psi \in \Psi, k \in \mathbb{Z}, \alpha \in \mathbb{Z}^{d}\right\},
$$

where

$$
E^{\alpha}: f \mapsto f(\cdot+\alpha)
$$

is the shift operator and

$$
D^{k}: f \mapsto|\operatorname{det} s|^{k / 2} f\left(s^{k} \cdot\right)
$$

is the dilation operator. A system $X \subset L_{2}\left(\mathbb{R}^{d}\right)$ is a fundamental tight frame with frame bound 1 if the map

$$
T^{*}: L_{2}\left(\mathbb{R}^{d}\right) \rightarrow \ell_{2}(X): f \mapsto(\langle f, x\rangle)_{x \in X}
$$

is unitary (but not necessarily onto: a tight frame with frame bound 1 whose corresponding $T^{*}$ is onto is necessarily orthonormal). In what follows, all systems that we treat are affine and fundamental, and all tight frames that are considered have frame bound 1, hence "a tight frame" should always be understood as "a fundamental tight affine frame with frame bound 1". A tight frame can be used for

Received by the editor February 19, 1996 and, in revised form, August 21, 1996.

1991 Mathematics Subject Classification. Primary 42C15 41A15 41A63; Secondary 42C30.

Key words and phrases. Affine systems, box splines, four-direction mesh, frames, tight frames, multiresolution analysis, wavelets .

This work was supported by the National Science Foundation under Grants DMS-9102857, DMS-9224748, and by the U.S. Army Research Office under Contracts DAAL03-G-90-0090, DAAH04-95-1-0089. 
the atomic decompositions of functions exactly in the same way orthonormal bases are used, i.e.,

$$
T^{*}: f \mapsto T^{*} f
$$

transform $f$ into discrete information, and its adjoint

$$
T: \ell_{2}(X) \rightarrow L_{2}: c \mapsto \sum_{x \in X} c(x) x
$$

can then be used to recover $f$ from its discrete transformation (i.e., $T T^{*} f=f$ ). In order for $T^{*}$ to exhibit good space-frequency localization, the functions $\Psi$ should be local in the space domain (ideally compactly supported), should be smooth (which leads to good decay in the frequency domain), and should provide positive approximation order. We refer to [D1], [D2], [HW] and [RS1], [RS2] for further discussions on frames and tight frames.

While tight frames should ideally be generated by few smooth compactly supported functions with simple structure, the only examples of compactly supported multivariate tight frames in the literature that we are aware of are bivariate orthonormal bases that were derived in $[\mathrm{CD}]$ from the univariate Daubechies' wavelets, [D3], as well as tensor products of Daubechies' wavelets. We briefly review those constructions now.

Let $L_{2}:=L_{2}\left(\mathbb{R}^{2}\right)$. Let $h$ be the refinement mask of a Daubechies' scaling function $\phi$, [D3] (whose shifts are known to be orthonormal), and let $\psi$ be the corresponding wavelet. Define the bivariate mask

$$
\tau\left(\omega_{1}, \omega_{2}\right):=h\left(\omega_{1}\right) .
$$

Given a dilation matrix $s$, a bivariate scaling function (or distribution) $\Phi$ can then be defined by

$$
\widehat{\Phi}: \omega \mapsto \prod_{j=1}^{\infty} \tau\left(s^{*-j} \omega\right) .
$$

Cohen and Daubechies employed in $[\mathrm{CD}]$ two different dilation matrices:

$$
s:=\left(\begin{array}{cc}
1 & 1 \\
1 & -1
\end{array}\right), \quad s_{1}:=\left(\begin{array}{cc}
1 & -1 \\
1 & 1
\end{array}\right),
$$

and obtained therefore two different scaling functions, say $\Phi$ and $\Phi_{1}$, respectively. They observed essential differences between the two so-obtained functions. The matrix $s$ satisfies $s^{2}=2 I$, and therefore the resulted $\Phi$ is clearly the tensor product (referred to as "separable" in $[\mathrm{CD}]$ )

$$
\Phi(x)=\phi\left(x_{2}\right) \phi\left(x_{1}-x_{2}\right),
$$

and therefore the shifts of $\Phi$ are necessarily orthonormal. Furthermore, the standard wavelet construction then yields the wavelet

$$
\Psi(x)=\psi\left(x_{2}\right) \phi\left(x_{1}-x_{2}\right) .
$$

While the refinable function $\Phi$ is separable, the refinable function $\Phi_{1}$ and the wavelet constructed from it are not separable in any sense. Nonetheless, it is proved in $[C D]$ that the shifts of $\Phi_{1}$ are orthonormal. It is further proved in [CD] that, 
unfortunately, $\Phi_{1}$ cannot be $C^{1}$, regardless of the order of the univariate scaling function which is used. Therefore, it is necessary to develop some other algorithms to construct nonseparable compactly supported tight frames with high smoothness.

It will not be entirely correct to say that the $[\mathrm{CD}]$ constructions and tensor product constructions comprise all known multivariate affine tight frames: according to [CS], tight frames can be constructed by appending to $\Psi$ some of their translates (=:oversampling); that, of course, only increases the number of elements in each of the above constructions, while preserving any deficiences (such as lack of symmetries and low smoothness in the non-separable case, and parallelogram supports in the separable case) that the orthonormal system may have had.

The theory established in [RS2], however, makes the construction of useful simple non-separable compactly supported tight frames with high smoothness and a variety of symmetries an easy task. In fact, one can essentially construct tight frames with the aid of the shifts of any refinable function, and therefore it is possible to impose a simple structure on the wavelets by selecting a refinable function with such desired structure. Indeed, we constructed in [RS2], for every positive integer $m$, a tight frame for $L_{2}(\mathbb{R})$ that is generated by $m$ wavelets, each of which is a spline of degree $m-1$, support $[0, m]$, and smoothness $C^{m-2}$. Further, all knots of the splinewavelets are half-integers, and each spline is either symmetric or anti-symmetric. (The case $m=4$ of this construction is discussed in $\S 4$ ).

Since the variety of possible constructions of multivariate (and univariate) tight frames based on the [RS2] theory is unlimited, we carefully selected for the present article those constructions which, in our opinion, may be used in practical applications. We kept in mind that different applications may require different properties from the wavelet system; for example, in data compression applications the number of different wavelets used (which accounts to the oversampling rate) should be minimized, while in finite element applications many elements with small supports may be preferred. Our two favorite bivariate constructions are detailed in $\S 2$ and $\S 3$ : in $\S 2$, the system is generated by many highly symmetric wavelets of small support (which, actually, are not so "many" for practical smoothness requirements); in $\S 3$, the system is generated by fewer larger elements. In each construction, the wavelets are splines, i.e., smooth piecewise-polynomials, always of compact support, with various symmetries. Moreover, the relevant grid is the four-direction (i.e., quincunx, see below) mesh, hence all the wavelets have a "round" octagonal support. On the other hand, similarly to our univariate construction, the number of elements used, as well as the volume of the support of each, increase together with the increase in the required smoothness. The ease in constructing tight spline frames is not limited to two dimensions; in $\S 4$ a general inductive algorithm for constructing multidimensional tight frames is provided. The algorithm works particularly well with box splines, in fact, with every box spline. We call the box spline wavelets obtained by this algorithm boxlets.

We have chosen to carry the constructions in $\S 2,3$ with respect to the dilation matrix $s$ (cf. 1.2). It is possible, though, to carry these same constructions with respect to $s_{1}$ with only one limitation: all four directions in the definition of the box spline must appear with the same multiplicity. In any event, the use of $s_{1}$ instead of $s$ does not yield different systems, and the reason is very simple: for a four-direction mesh, not only that $s \mathbb{Z}^{2}=s_{1} \mathbb{Z}^{2}$, but also, in case the box spline $\phi$ has equi-multiplicities, $\phi(s \cdot)=\phi\left(s_{1} \cdot\right)$. 


\section{Bivariate TIGHT FRAMES I: GENERATORS OF SMALL SUPPORT}

We construct here, for any integer $m>1$, bivariate spline tight frames generated by $m^{2}-1$ mother wavelets in $C^{3 m-5}$, as well as tight frames generated by $m^{2}+m-1$ wavelets of smoothness $C^{3 m-4}$.

Let $\phi$ be the box spline

$$
\widehat{\phi}(\omega)=\prod_{j=1}^{4}\left(\frac{1-e^{-i \xi_{j} \cdot \omega}}{i \xi_{j} \cdot \omega}\right)^{m_{j}},
$$

where

$$
\left(\xi_{1}, \xi_{2}, \xi_{3}, \xi_{4}\right):=\left(\left(\begin{array}{l}
1 \\
0
\end{array}\right) \quad\left(\begin{array}{l}
0 \\
1
\end{array}\right) \quad\left(\begin{array}{l}
1 \\
1
\end{array}\right) \quad\left(\begin{array}{c}
1 \\
-1
\end{array}\right)\right),
$$

and with $m_{1}=m_{3}$, and $m_{2}=m_{4}$. It is well-known (cf. [BHR]) that the box spline $\phi$ satisfies the following properties:

(a) It is a piecewise-polynomial of local degree $2\left(m_{1}+m_{2}-1\right)$, on the fourdirection mesh (that is obtained by adding the diagonals to each square of integer vertices).

(b) It is globally $C^{\rho-2}$ with $\rho:=\min \left\{2 m_{1}+m_{2}, 2 m_{2}+m_{1}\right\}$; (in fact, its $(\rho-2)$ -

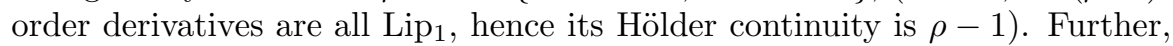
it provides approximation order $\rho$.

(c) It is supported in the octagon

$$
\left\{\sum_{j=1}^{4} t_{j} \xi_{j}: 0 \leq t_{j} \leq m_{j}, j=1, \ldots, 4\right\} .
$$

Further, it is essentially positive on its support.

In the sequel, we will introduce a variety of octagonal domains similar to the support of $\phi$ above. For reasons of efficiency, we therefore denote

$$
\left[a_{1}, a_{2}, a_{3}, a_{4}\right]:=\left\{\sum_{j=1}^{4} t_{j} \xi_{j}: 0 \leq t_{j} \leq a_{j}, j=1, \ldots, 4\right\} .
$$

Note that each $[a, b, c, d]$ is an octagon whose area is

$$
\operatorname{area}([a, b, c, d])=a b+a c+a d+b c+b d+2 c d .
$$

We prefer the above box spline over other variants because it is refinable not only with respect to the dilation matrix $2 I$, but also with respect to the dilation matrix

$$
s:=\left(\begin{array}{cc}
1 & 1 \\
1 & -1
\end{array}\right)
$$

This well-known fact was rarely exploited before because the shifts of the 4-direction box spline do not form a Riesz basis (or a frame). The only construction of compactly supported four-directional spline frames that we are aware of, appears in [CJS]. There, the same dilation matrix $s$ is employed, and a compactly supported spline wavelet that is orthogonal to the box spline space while lying in the next dilation level is identified. This wavelet is then complemented by one of its translates, and the affine system is then generated by these two mother wavelets, and is proved, by an involved argument, to be a frame. The resulted frame is not tight, and no estimates for the frame bounds are given in [CJS]. In fact, the problem of 
finding a dual frame is not addressed in [CJS], and it is likely that a dual frame of compact support does not exist.

In contrast, our constructions of tight frames are obtained with relative ease. The main reason for that is that the [RS2] theory provides us with a simple criterion for tight frames, that does not impose any a priori assumption on the refinable function $\phi$.

A straightforward computation shows that

$$
\widehat{\phi}(s \cdot)=\tau_{0} \widehat{\phi}
$$

with the function

$$
\tau_{0}(\omega):=\left(\frac{1+e^{-i \omega_{1}}}{2}\right)^{m_{1}}\left(\frac{1+e^{-i \omega_{2}}}{2}\right)^{m_{2}}=\theta(\omega) \cos ^{m_{1}}\left(\omega_{1} / 2\right) \cos ^{m_{2}}\left(\omega_{2} / 2\right),
$$

with

$$
\theta(\omega):=e^{-i\left(m_{1} \omega_{1}+m_{2} \omega_{2}\right) / 2} .
$$

We note that $\phi$ is refinable, which means that the mask $\tau_{0}$ is $2 \pi$-periodic (and hence $\phi\left(s^{-1}\right.$.) can be written as a linear combination of the integer translates of $\left.\phi\right)$.

Our construction invokes the following theorem, which is a special case of Corollary 6.7 of [RS2].

Theorem 2.3. Let $\phi$ be any box spline that is refinable with respect to the dilation

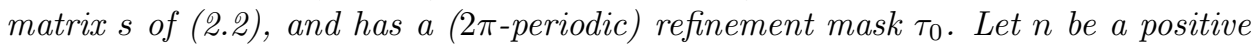
integer, and let $\left(\tau_{j}\right)_{j=1}^{n}$ be $n 2 \pi$-periodic essentially bounded measurable functions. Assume that, for a.e. $\omega$, and for $\nu:=(\pi, \pi)$,

$$
\sum_{j=0}^{n}\left|\tau_{j}\right|^{2}=1, \quad \sum_{j=0}^{n} \tau_{j} \overline{E^{\nu} \tau_{j}}=0 .
$$

Then the wavelets $\Psi:=\left(\psi_{j}\right)_{j=1}^{n}$, defined by

$$
\widehat{\psi}(s \cdot):=\tau_{j} \widehat{\phi}, \quad j=1, \ldots, n,
$$

generate a tight frame (i.e., a fundamental affine tight frame with frame bound 1) for $L_{2}$.

It should be understood that the dilation matrix $s$ that is involved in the refinement equation is the same dilation matrix $s$ that is used to generate the affine system from $\Psi$.

To construct now the wavelets with the aid of the above box spline $\phi$, we first define the following univariate $4 \pi$-periodic functions:

$$
y_{j}(t):=y_{j}^{[m]}(t):=\sqrt{\left(\begin{array}{c}
m \\
j
\end{array}\right)} \cos ^{m-j} t / 2 \sin ^{j} t / 2,0 \leq j \leq m .
$$

(Warning: $m$ in $y_{j}^{[m]}$ is an index, not a power!) Note that, for any fixed $m$,

$$
\sum_{j=0}^{m}\left|y_{j}\right|^{2}=1, \quad \sum_{j=0}^{m} y_{j} \overline{y_{j}(\cdot+\pi)}=0 .
$$

We next define the tensor product bidimensional mask system

$$
\left(\tau_{n}: n \in N:=\left\{0,1, \ldots, m_{1}\right\} \times\left\{0,1, \ldots, m_{2}\right\}\right)
$$


with

$$
\tau_{n}(\omega):=\theta(\omega) y_{n_{1}}^{\left[m_{1}\right]}\left(\omega_{1}\right) y_{n_{2}}^{\left[m_{2}\right]}\left(\omega_{2}\right) .
$$

Each $\tau_{n}$ is $2 \pi$-periodic.

We then observe that $\tau_{0}:=\tau_{0,0}$ in the above tensor system is the refinement mask of $\phi$. Moreover, the convex hull of the spectrum of each $\tau_{n}, n \in N$, is independent of $n$ (i.e., in down-to-earth language: the $2 \pi$-integers that lie in the convex hull of the frequencies of the exponentials whose linear combination form $\tau_{n}$, are the same for all $n)$. This means that the $\left(m_{1}+1\right)\left(m_{2}+1\right)-1$ functions, defined by

$$
\Psi:=\left\{\psi_{n}: \widehat{\psi}_{n}(s \cdot):=\tau_{n} \widehat{\phi}, \quad n \in N \backslash 0\right\},
$$

are all supported in the support of $\phi$, i.e., in the octagon $\left[m_{1}, m_{2}, m_{1}, m_{2}\right]$. The functions $\Psi$ are non-separable piecewise-polynomials, with the same smoothness as the box spline $\phi$ used.

The tensor product structure of the masks (together with the fact that $|\theta|=1$ ) clearly implies (cf. (2.5)) that

$$
\sum_{n \in N}\left|\tau_{n}(\omega)\right|^{2}=\left(\sum_{j=0}^{m_{1}}\left|y_{j}^{\left[m_{1}\right]}\left(\omega_{1}\right)\right|^{2}\right)\left(\sum_{j=0}^{m_{2}}\left|y_{j}^{\left[m_{2}\right]}\left(\omega_{2}\right)\right|^{2}\right)=1,
$$

and by the same token, for $\nu=(\pi, \pi)$ (with $c:=\theta \overline{E^{\nu} \theta}$ )

$$
\begin{aligned}
& \sum_{n \in N} \tau_{n}(\omega) \overline{E^{\nu} \tau_{n}(\omega)} \\
& \quad=c\left(\sum_{j=0}^{m_{1}} y_{j}^{\left[m_{1}\right]}\left(\omega_{1}\right) \overline{y_{j}^{\left[m_{1}\right]}\left(\omega_{1}+\pi\right)}\right)\left(\sum_{j=0}^{m_{2}} y_{j}^{\left[m_{2}\right]}\left(\omega_{2}\right) \overline{y_{j}^{\left[m_{2}\right]}\left(\omega_{2}+\pi\right)}\right)=0 \cdot 0=0 .
\end{aligned}
$$

Therefore, Theorem 2.3 implies the following:

Theorem 2.7. The wavelets $\Psi$ constructed in (2.6) generate a tight affine frame with frame bound 1 .

Remark. Each one of these wavelets is supported in the support of $\phi$, and is a $C^{\rho-2}$, $\rho:=\min \left\{2 m_{1}+m_{2}, 2 m_{2}+m_{1}\right\}$, piecewise-polynomial of local degree $2\left(m_{1}+m_{2}-1\right)$, with respect to the mesh $s^{-1} M$, with $M$ the standard four-direction mesh. The mesh $s^{-1} M$ consists of all lines of the form

$$
x_{1}+x_{2}=j, x_{1}-x_{2}=j, x_{1}=j / 2, x_{2}=j / 2,
$$

where $j$ varies over $\mathbb{Z}$, and $\left(x_{1}, x_{2}\right)$ is the generic point in the space domain.

Example. We take $m_{1}=m_{2}=1$. In this case $\phi$ is the well-known $C^{1}$ piecewisequadratic Zwart element, supported in the octagon $[1,1,1,1]$. The above construction yields three $C^{1}$ piecewise-quadratic wavelets each supported in $[1,1,1,1]$, and each exhibiting various symmetries (cf. Figures 2.1 and 2.2). The support of each wavelet is of area 7 ; this small support comes despite of the fact that we oversample by a factor of 3 .

It is hard to compare the above construction to literature counterparts, since, as we mentioned before, the latter hardly exist. Here are three possible comparisons. In the first, we take the separable construction of [CD], using Daubechies scaling 


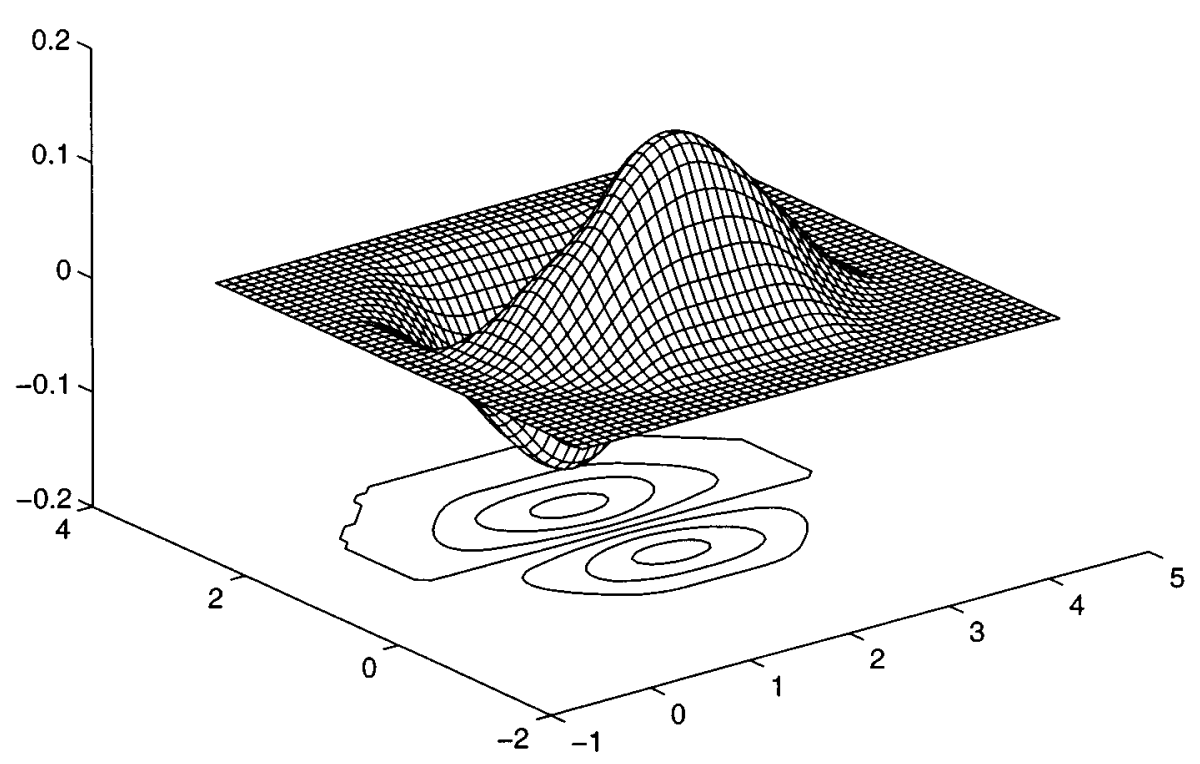

Figure 2.1. The $C^{1}$ piecewise-quadratic wavelet $\psi_{0,1}$. The wavelet $\psi_{1.0}$ is obtained by rotation.

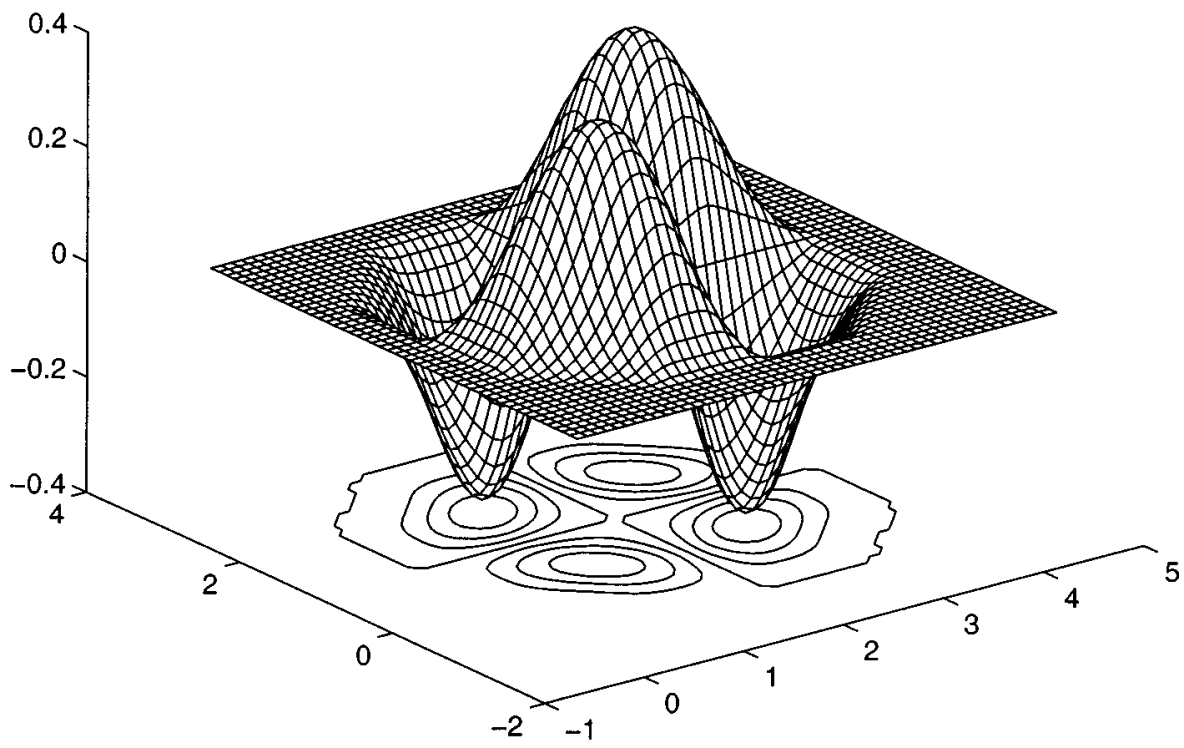

Figure 2.2. The $C^{1}$ piecewise-quadratic wavelet $\psi_{1,1}$. Note the octagonal support.

function that provides the same approximation order (i.e., 3) as the Zwart element. The result is a single wavelet supported in a parallelogram of area 25, and whose shifts are orthonormal. The smoothness of this half of the above tight frame (in terms of Hölder exponents: 2 vs. 1.1; cf. [D2, pp. 232-239]), and it lacks symmetries. 
Another comparison may be with "plain" tensor product of the same Daubechies' scaling function. In this case, we obtain 3 mother wavelets each with a square support of area 25, and with the same smoothness and (lack of) symmetries. The shifts of these wavelets now fill an entire dyadic level. To fill in an entire dyadic level using integer shifts of our elements, we need the three elements of support area of 7 each, and 6 elements of half-size support, with total area of supports 42 .

Finally, the [CJS] construction provides here two smooth symmetric spline wavelets each supported in a domain of area 28. However, as mentioned before, this frame is not tight, and no simple recipe for a dual frame is known.

Example. We take $m_{1}=1, m_{2}=2$. In this case $\phi$ is globally $C^{2}$ (and is $C^{3}$ on half of the mesh lines), piecewise-quartic. The construction now yields 5 wavelets, all supported in the octagon $[1,2,1,2]$ whose area is 15 . They all have the same (global) smoothness and local degree as the box spline.

Example 2.8. We take $m_{1}=m_{2}=2$. Then we obtain 8 elements supported each in the octagon $[2,2,2,2]$ whose vertices (up to a $(1,1)$-shift) are $( \pm 3, \pm 1) \cup( \pm 1, \pm 3$ ) and whose area is 28. Each spline wavelet is $C^{4}$ (with Hölder exponent 5). The local polynomial degree is 6 .

\section{Bivariate Tight FRAMES II: FEWER GENERATORS}

Here, for any integer $m$, we construct tight frames generated by either $2 m$ mother wavelets in $C^{3 m-2}$, or $2 m+1$ wavelets in $C^{3 m-1}$.

The wavelets constructed in this section are selected from the same box spline spaces used in the previous section. Given a four-direction box spline with corresponding multiplicities $\left(m_{1}, m_{2}, m_{1}, m_{2}\right)$, the construction selects appropriate mother wavelets as the $s$-dilate of certain functions in the span of the shifts of the box spline. This means that the wavelets here are comparable to the wavelets of the previous section in terms of local polynomial degrees, underlying meshes, and smoothness.

However, the number of wavelets associated with the previous construction grows quadratically with the required smoothness. Though the three examples that followed show that for practical smoothness requirements the number of wavelets is "within reason", it is possible to construct tight spline frames whose number of generators grows only linearly with the required smoothness. The associated wavelets, on the other hand, have larger, somewhat less symmetric, support.

It is convenient to carry out the construction here not with the aid of the box spline $\phi$ of (2.1), but, rather, with the following "averaged" spline $\varphi$ :

$$
\widehat{\varphi}(\omega):=\left(\frac{1+e^{-i \omega_{1}}}{2}\right)^{m_{1}} \widehat{\phi}(\omega)
$$

In standard box spline terminology, $\varphi$ is a box spline with direction set $\left(2 \xi_{1}, \xi_{2} \xi_{3}, \xi_{4}\right)$ (cf. (2.1)) with corresponding multiplicities $\left(m_{1}, m_{2}, m_{1}, m_{2}\right)$. Also, direct computation yields that $\widehat{\varphi}$ satisfies

$$
\begin{aligned}
\widehat{\varphi}(s \omega) & =\left(\frac{1+e^{-i\left(\omega_{1}+\omega_{2}\right)}}{2}\right)^{m_{1}}\left(\frac{1+e^{-i \omega_{2}}}{2}\right)^{m_{2}} \widehat{\varphi}(\omega) \\
& =\vartheta(\omega) \cos ^{m_{1}}\left(\left(\omega_{1}+\omega_{2}\right) / 2\right) \cos ^{m_{2}}\left(\omega_{2} / 2\right) \widehat{\varphi}(\omega),
\end{aligned}
$$


where

$$
\vartheta(\omega):=e^{-i\left(m_{1}, m_{1}+m_{2}\right) \cdot \omega / 2}
$$

Recalling now the definition of $y_{j}^{[m]}$ in (2.4), we define $m_{1}+m_{2}+12 \pi$-periodic functions as follows:

$$
\tau_{j}(\omega):=\vartheta(\omega) y_{j}^{\left[m_{1}\right]}\left(\omega_{1}+\omega_{2}\right) y_{0}^{\left[m_{2}\right]}\left(\omega_{2}\right), \quad j=0, \ldots, m_{1},
$$

and

$$
\tau_{m_{1}+j}(\omega):=\theta(\omega) y_{j}^{\left[m_{2}\right]}\left(\omega_{2}\right), \quad j=1, \ldots, m_{2},
$$

where

$$
\theta(\omega):=e^{-i m_{2} \omega_{2} / 2}
$$

We note that the functions $e^{-i m_{1}\left(\omega_{1}+\omega_{2}\right) / 2} y_{j}^{\left[m_{1}\right]}\left(\omega_{1}+\omega_{2}\right)$ are periodic with respect to shifting by $(\pi, \pi)$, hence are periodic with respect to the lattice $s^{-1} 2 \pi \mathbb{Z}^{2}$. From that, together with the fact, $(2.5)$, that

$$
\sum_{j=0}^{m_{1}}\left|y_{j}^{\left[m_{1}\right]}\right|^{2}=1
$$

one immediately concludes that for $\varepsilon \in\{0,1\}$, and $\nu=(\pi, \pi)$,

$$
\sum_{j=0}^{m_{1}} \tau_{j}(\omega) \overline{E^{\varepsilon \nu} \tau_{j}(\omega)}=\theta(-\varepsilon \pi) y_{0}^{\left[m_{2}\right]}\left(\omega_{2}\right) \overline{y_{0}^{\left[m_{2}\right]}\left(\omega_{2}+\varepsilon \pi\right)} .
$$

This leads further to the conclusions that, firstly,

$$
\sum_{j=0}^{m_{1}+m_{2}}\left|\tau_{j}\right|^{2}=\sum_{j=0}^{m_{2}}\left|y_{j}^{\left[m_{2}\right]}\right|^{2}=1,
$$

and, secondly, with $\nu:=(\pi, \pi)$,

$$
\sum_{j=0}^{m_{1}+m_{2}} \tau_{j}(\omega) \overline{E^{\nu} \tau_{j}}(\omega)=\theta(-\pi) \sum_{j=0}^{m_{2}} y_{j}^{\left[m_{2}\right]}\left(\omega_{2}\right) \overline{y_{j}^{\left[m_{2}\right]}\left(\omega_{2}+\pi\right)}=0 .
$$

In summary, the mask vector

$$
\left(\tau_{j}\right)_{j=0}^{m_{1}+m_{2}}
$$

satisfies the conditions of Theorem 2.3.

Consequently, since $\tau_{0}$ in the above construction is the refinement mask of $\varphi$, we conclude from Theorem 2.3 that the $m_{1}+m_{2}$ wavelets defined by

$$
\widehat{\psi}_{j}(s \cdot):=\tau_{j} \widehat{\varphi}, \quad j=1, \ldots, m_{1}+m_{2},
$$

generate a tight frame.

Corollary 3.1. Let $\varphi$ be the box spline whose direction set is $\left(2 \xi_{1}, \xi_{2}, \xi_{3}, \xi_{4}\right)$ with corresponding multiplicities $\left(m_{1}, m_{2}, m_{1}, m_{2}\right)$ :

$$
\widehat{\varphi}=\left(\frac{1+e^{-i \omega_{1}}}{2}\right)^{m_{1}} \widehat{\phi}(\omega)
$$

with $\phi$ the box spline of (2.1). Then, with $\left(\tau_{j}\right)_{j=1}^{m_{1}+m_{2}}$ the above constructed masks, the wavelets $\Psi:=\left(\psi_{j}\right)_{j=1}^{m_{1}+m_{2}}$, defined by $\widehat{\psi}_{j}(s \cdot):=\tau_{j} \widehat{\varphi}$, generate a tight frame (with frame bound 1) for $L_{2}$. These wavelets share the same smoothness, local degree, and mesh with the wavelets of Theorem 2.7. 


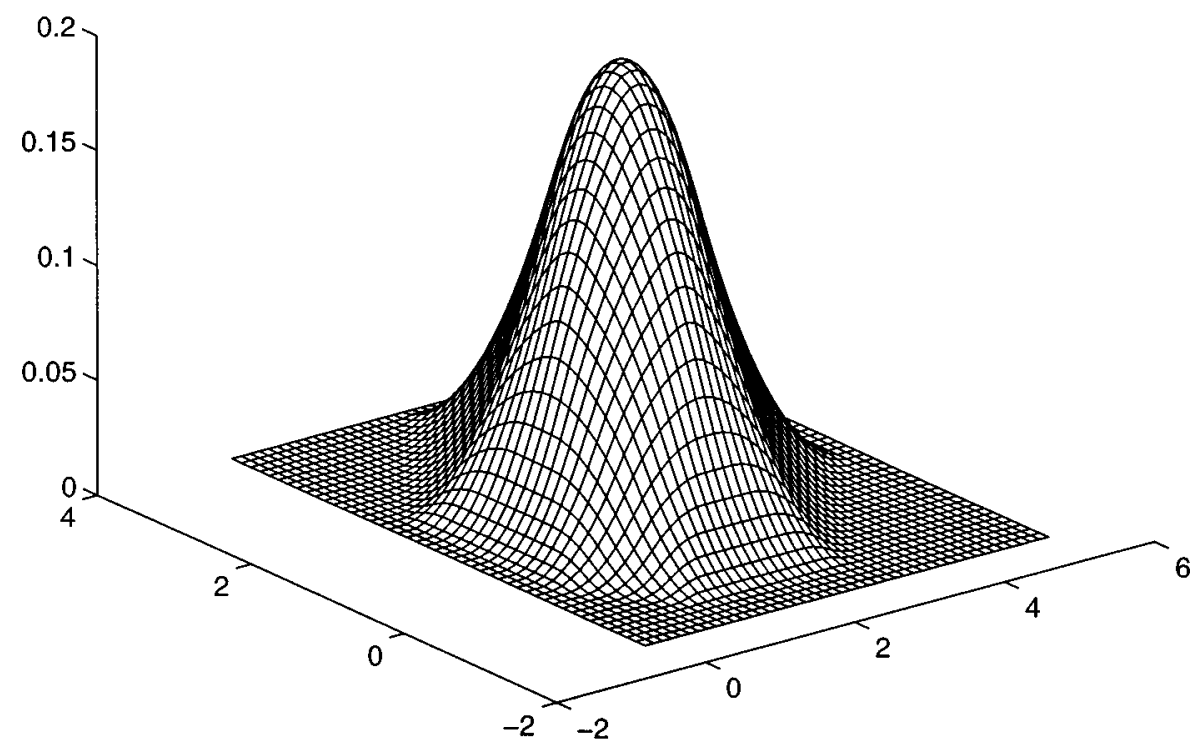

Figure 3.1. The $C^{1}$ piecewise-quadratic box spline that is used in Example 3.2.

Discussion. The box spline $\varphi$ in the above corollary is supported in the octagon $\left[2 m_{1}, m_{2}, m_{1}, m_{2}\right]$ whose area is $2 m_{1}^{2}+7 m_{1} m_{2}+m_{2}^{2}$. As to the wavelets, the "large" wavelets, i.e., the first $m_{1}$ elements, have the same support as $\varphi$. The other $m_{2}$ elements have the octagonal support $\left[m_{1}, m_{2}, m_{1}, m_{2}\right]$, whose area is $m_{1}^{2}+5 m_{1} m_{2}+$ $m_{2}^{2}$.

Remark. The general construction detailed in the next section shows that there are many possible modifications of the above construction.

Example 3.2. We consider the case $m_{1}=m_{2}=1$. Figure 3.1 shows the box spline $\varphi$. It is a $C^{1}$ piecewise-quadratic that provides approximation order 3 , and it is supported in the octagon $[2,1,1,1]$, with area 10 . Two wavelets are constructed here. Direct computation yields that the larger support wavelet, $\psi_{1}$ (cf. Figure 3.2), whose support is identical to that of $\varphi$, satisfies

$$
\widehat{\psi}_{1}(\omega)=\tan \left(\omega_{1} / 2\right) \widehat{\varphi}(\omega),
$$

while the smaller support $\psi_{2}$ (cf. Figure 3.3) has the form

$$
\widehat{\psi}_{2}=\frac{1-e^{-i\left(\omega_{1}-\omega_{2}\right) / 2}}{2 i} \widehat{\varphi}\left(\frac{\omega_{1}+\omega_{2}}{2}, \frac{\omega_{1}-\omega_{2}}{2}\right) .
$$

The corresponding supports are $[2,1,1,1]$ and $[1,1,1,1]$ with corresponding areas 10 and 7.

Example. We consider $m_{1}=1, m_{2}=2$. The box spline $\varphi$ is then $C^{2}$ piecewisequartic and provides approximation order 4 . Its support is the octagon $[2,2,1,2]$ whose area is 20 . The mother wavelet set consists of three elements, two "small" and one "large". The large element $\psi_{1}$ is defined exactly as in (3.3) (only that $\varphi$ 


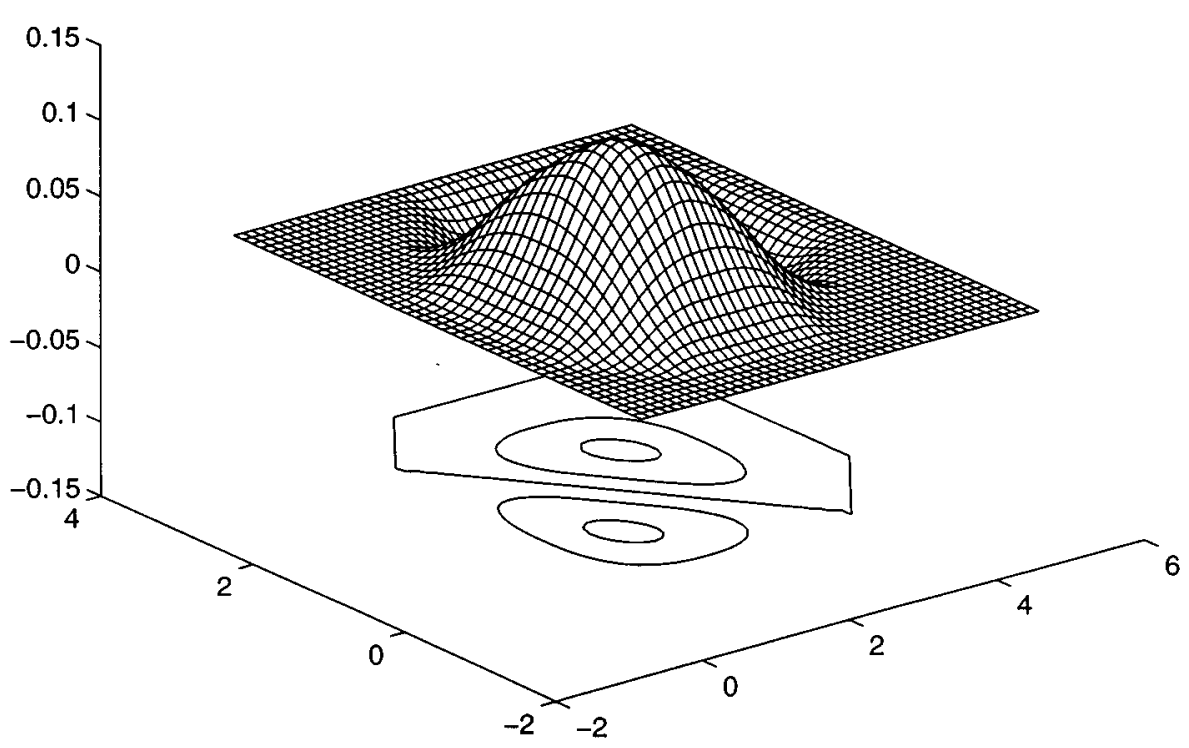

Figure 3.2. The $C^{1}$ piecewise-quadratic wavelet with larger support $\psi_{1}$.

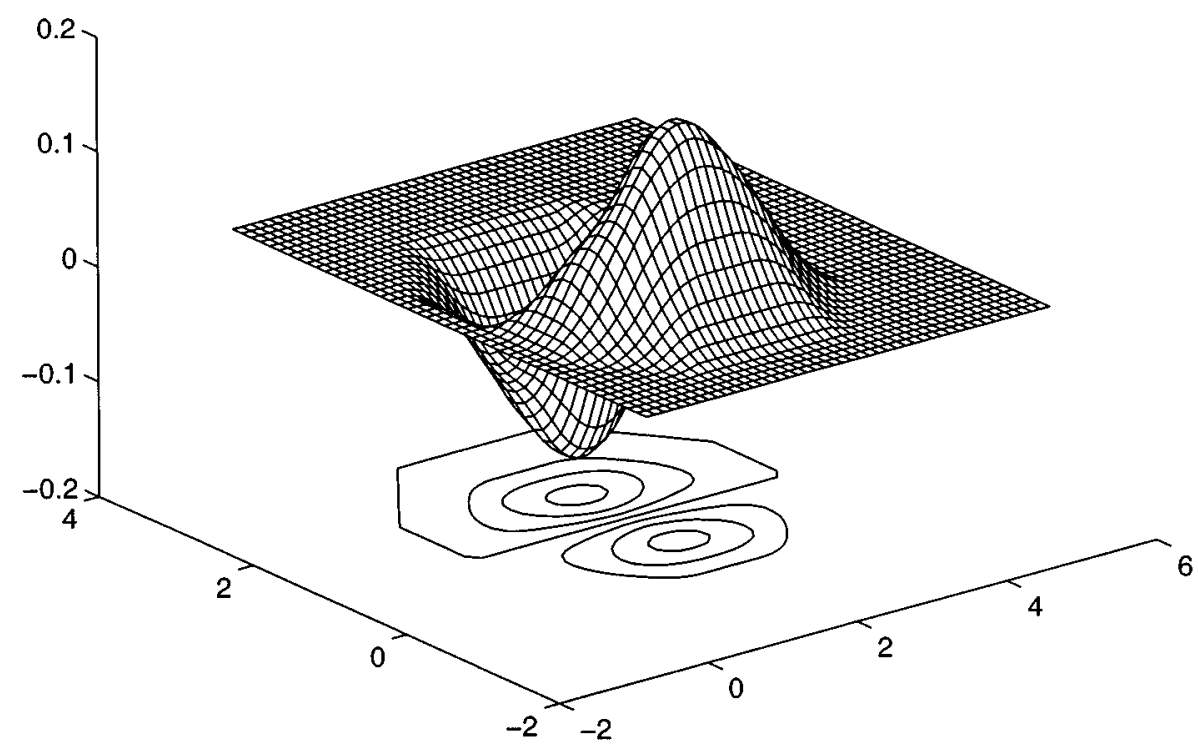

Figure 3.3. The $C^{1}$ piecewise-quadratic wavelet with smaller support $\psi_{2}$.

has been changed), and has the support of $\phi$. The other wavelets are

$$
\widehat{\psi}_{2}=\frac{1-e^{-i\left(\omega_{1}-\omega_{2}\right)}}{2 \sqrt{2} i} \widehat{\varphi}\left(\frac{\omega_{1}+\omega_{2}}{2}, \frac{\omega_{1}-\omega_{2}}{2}\right),
$$


and

$$
\widehat{\psi}_{3}=\frac{-1+2 e^{-i \frac{\omega_{1}-\omega_{2}}{2}}-e^{-i\left(\omega_{1}-\omega_{2}\right)}}{4} \widehat{\varphi}\left(\frac{\omega_{1}+\omega_{2}}{2}, \frac{\omega_{1}-\omega_{2}}{2}\right),
$$

both supported in the same octagon $[1,2,1,2]$, whose area is 15 .

Example. The case $m_{1}=m_{2}=2$ yields four wavelets, two supported in $[4,2,2,2]$, and the other two supported in $[2,2,2,2]$. The area of these octagons is 40 and 28 respectively, hence their total area is 136 . The smoothness of the box spline, as well as its local degree, is the same as in Example 2.8.

\section{A General induCtive ALGORITHM FOR CONSTRUCTING TIGHT FRAMES}

The method described in the last section for the construction of box spline wavelets on a four-direction mesh can be significantly generalized. Since we are unable to predict, at the time when this article is written, what specific variants may be implemented in practice, we decided to simply outline the highest level of generalization that we are able to observe.

The setup here is as follows: we hold a dilation matrix $s$ (i.e., an integer matrix whose inverse is contractive) and two $d$-variate functions (or, more generally, distributions), say $\phi_{1}, \phi_{2}$, that are refinable with respect to $s$. The corresponding masks, that are assumed to be $2 \pi$-periodic (that is a part of the definition of refinability), are also assumed to be bounded; in practical situations the masks are trigonometric polynomials, hence certainly bounded. The basic assumption is that we already know how to derive a tight frame from the scaling function $\phi_{1}$, and we would like to use that known frame in order to obtain a new, improved (in terms of smoothness, for example) tight frame. Specifically, we would like to extract the new frame from the (necessarily refinable) convolution

$$
\phi:=\phi_{1} * \phi_{2} .
$$

This idea (of convolving the given scaling function with a suitably chosen distribution) has been used in the spline and wavelet theory many times. For example, Daubechies obtained her univariate refinable functions whose shifts are orthonormal by convolving a univariate B-spline $\phi_{1}$ (whose shifts are stable but not orthonormal), with a suitably chosen distribution $\phi_{2}$. In her construction, the new $\phi$ provides the same approximation order as the B-spline $\phi_{1}$, but is significantly less smooth; (the smoothness of the scaling function and the orthonormality of its shifts are then transferred to the constructed wavelets). The fact that one cannot obtain by convolution the orthonormality of the shifts, while simultaneously improving, or at least retaining, the smoothness of the original $\phi_{1}$, is one of the main reasons the construction of affine orthonormal bases is fairly involved.

The theory of [RS2] allows us to pay less attention to the properties of the scaling function: "bad" properties of the latter may not at all be inherited by the wavelets! Specifically, the smoothness of the wavelets and the tightness of the frame they generate do not compete any more. In fact, we can separate the construction of the smooth tight frame into two steps: in the first a basic low-smoothness frame is constructed, and then the smoothness of the frame is improved without hampering its tightness: in terms of $\phi_{1}, \phi_{2}$ above, $\phi_{1}$ is the low- (or intermediate-) smooth scaling function that yields a tight affine system, and $\phi_{2}$ is the convolutor that should improve the smoothness. 
We specify now the exact conditions that are imposed on $\phi_{1}, \phi_{2}$. In these conditions, we use the notation

$$
\Gamma:=2 \pi\left(s^{*-1} \mathbb{Z}^{d} / \mathbb{Z}^{d}\right) .
$$

For example, if $s=2 I, \Gamma$ is the group $\{0, \pi\}^{d}$, with addition modulo $2 \pi$. Note that the order of $\Gamma$ is $|\operatorname{det} s|$.

(4.1) Conditions assumed on $\phi_{1}, \phi_{2}$.

(a) For $j=1,2, \phi_{j}$ is a refinable distribution with bounded mask $\tau_{j}$.

(b) There exists a collection $\mathrm{T}_{1}$ of $2 \pi$-periodic bounded functions that satisfy, for every $\nu \in \Gamma$, and a.e. on $\mathbb{R}^{d}$,

$$
\tau_{1} \overline{E^{\nu} \tau_{1}}+\sum_{\tau \in \mathrm{T}_{1}} \tau \overline{E^{\nu} \tau}=\delta_{\nu}
$$

(c) There exist another collection of $2 \pi$-periodic bounded functions $\mathrm{T}_{2}$, that satisfy

$$
\left|\tau_{2}\right|^{2}+\sum_{\tau \in \mathrm{T}_{2}}|\tau|^{2}=1
$$

Discussion. The fact that we assume $\phi_{2}$ to be merely a distribution seems to be practically important as we will see in the examples presented at the end of this section. On the other hand, $\phi_{1}$, in all examples we carry in mind, is a function. In fact, if $\phi_{1}$ is a function, Corollary 6.7 of [RS2] "almost" implies that, under the condition (b) of the conditions (4.1), the system generated by the wavelets that are defined via their Fourier transforms by

$$
\left\{\widehat{\psi}\left(s^{*} \cdot\right):=\tau \widehat{\phi}_{1}: \tau \in \mathrm{T}_{1}\right\}
$$

is a tight frame for $L_{2}\left(\mathbb{R}^{d}\right)$. The only missing condition is a very mild smoothness requirement of $\phi_{1}$, that all box splines, for example, satisfy (cf. (4.6) of [RS2]). Note that significantly weaker conditions are assumed on $T_{2}$.

With the assumptions of (4.1) in hand, we will be able to construct a tight frame based not on the convolution $\phi_{1} * \phi_{2}$, but, rather, on the convolution with larger support

$$
\phi:=\frac{\phi_{2}\left(s^{-1} \cdot\right) * \phi_{1}}{|\operatorname{det} s|} .
$$

Note that $\widehat{\phi}=\widehat{\phi}_{2}\left(s^{*}\right) \widehat{\phi}_{1}$, and hence that $\phi$ is refinable with mask

$$
\tau_{\phi}:=\tau_{2}\left(s^{*} \cdot\right) \tau_{1}
$$

We now introduce the new mask collection

$$
\mathrm{T}:=\mathrm{T}_{1} \cup\left(\tau_{1} \mathrm{~T}_{2}\left(s^{*} \cdot\right)\right),
$$

i.e., we apply $s^{*}$ dilation to each of the masks in $\mathrm{T}_{2}$, multiply each by $\tau_{1}$, and then append the new collection to $\mathrm{T}_{1}$. Clearly,

$$
\# \mathrm{~T}=\# \mathrm{~T}_{1}+\# \mathrm{~T}_{2} \text {. }
$$

Lemma 4.2. Each of the masks in $\mathrm{T}$ above is $2 \pi$-periodic and bounded. Furthermore, for every $\nu \in \Gamma$,

$$
\tau_{\phi} \overline{E^{\nu} \tau_{\phi}}+\sum_{\tau \in T} \tau \overline{E^{\nu} \tau}=\delta_{\nu}
$$


Proof. We note that the masks in $\mathrm{T}_{2}\left(s^{*}\right)$ are not only $2 \pi \mathbb{Z}^{d}$-periodic, but also $2 \pi s^{*-1} \mathbb{Z}^{d}$-periodic. Therefore, for every $\nu \in \Gamma$, by (c) of (4.1),

$$
\tau_{\phi} \overline{E^{\nu} \tau_{\phi}}+\sum_{\tau \in \mathrm{T}_{2}\left(s^{*}\right)}\left(\tau_{1} \tau\right) \overline{E^{\nu}\left(\tau_{1} \tau\right)}=\left(\tau_{1} \overline{E^{\nu} \tau_{1}}\right)\left(\left|\tau_{2}\left(s^{*} \cdot\right)\right|^{2}+\sum_{\tau \in \mathrm{T}_{2}\left(s^{*}\right)}|\tau|^{2}\right)=\tau_{1} \overline{E^{\nu} \tau_{1}} .
$$

This implies that, by (b) of (4.1), for every $\nu \in \Gamma$,

$$
\tau_{\phi} \overline{E^{\nu} \tau_{\phi}}+\sum_{\tau \in \mathrm{T}} \tau \overline{E^{\nu} \tau}=\tau_{1} \overline{E^{\nu} \tau_{1}}+\sum_{\tau \in \mathrm{T}_{1}} \tau \overline{E^{\nu} \tau}=\delta_{\nu}
$$

As we already mentioned above, the properties proved with respect to $\mathrm{T}$ in the above lemma almost imply that the wavelet system $\Psi:=(\psi)_{\psi \in \Psi}$ defined by

$$
\left\{\widehat{\psi}\left(s^{*} \cdot\right):=\tau \widehat{\phi}: \tau \in \mathrm{T}\right\}
$$

generates a tight frame: we only need a mild smoothness condition of $\phi$. Rather than quoting the complicated condition (4.6) of [RS2], we assume in the following corollary smoothness conditions that are slightly stronger but more transparent.

Corollary 4.3. In the above notations, if the refinable function $\phi$ is a box spline, or if its Fourier transform decays at $\infty$ like $O\left(|\cdot|^{-1 / 2-\delta}\right)$ for $\delta>0$, then the wavelets $\Psi$ constructed as above generate a fundamental affine tight frame with frame bound 1 for $L_{2}\left(\mathbb{R}^{d}\right)$.

(4.4) Boxlets. We assume that the dilation matrix $s$ satisfies $s^{k}=2 I$, for some positive integer $k$. In this case, there is a natural way for choosing the smoothing factor $\phi_{2}$ : starting with a cycle $\Xi:=\left(\xi_{1}, \ldots, \xi_{k}\right)$ of $s$ (i.e., $s \xi_{j}=\xi_{j+1}, j=$ $1, \ldots, k-1$, hence necessarily $s \xi_{k}=2 \xi_{1}$ ), we define $\phi_{2}$ as the box spline with directions $\Xi$ :

$$
\widehat{\phi}_{2}(\omega)=\prod_{\xi \in \Xi} \frac{1-e^{-i \xi \cdot \omega}}{i \xi \cdot \omega} .
$$

Then, $\phi_{2}$ is refinable with mask

$$
\tau_{2}(\omega)=\frac{1+e^{-i \xi_{1} \cdot \omega}}{2} .
$$

Since we tacitly assume $\Xi$ to be integer, $\tau_{2}$ is $2 \pi$-periodic. Selecting $T_{2}$ is then trivial: $\mathrm{T}_{2}$ is taken to be the singleton

$$
\mathrm{T}_{2}:=\left\{\frac{1-e^{-i \xi_{1} \cdot \omega}}{2}\right\} .
$$

The satisfaction of (c) in (4.1) is then automatic, and, assuming that $\mathrm{T}_{1}, \phi_{1}$ are already given, and that the refinement mask of $\phi_{1}$ is $\tau_{1}$, our general inductive step reads here as follows:

(a) The new refinable function is $\phi:=\frac{\phi_{1} * \phi_{2}\left(s^{-1} \cdot\right)}{|\operatorname{det} s|}$.

(b) The refinement mask of $\phi$ is

$$
\tau_{\phi}(\omega):=\tau_{1}(\omega) \frac{1+e^{-i s \xi_{1} \cdot \omega}}{2}=\tau_{1}(\omega) \frac{1+e^{-i \xi_{2} \cdot \omega}}{2} .
$$

(c) The new wavelet mask set $\mathrm{T}$ is obtained by appending to $\mathrm{T}_{1}$ the single mask

$$
\tau(\omega):=\tau_{1}(\omega) \frac{1-e^{-i \xi_{2} \cdot \omega}}{2} .
$$


Boxlets correspond to the choice $s=2 I$ for the dilation matrix. The cycles of $2 I$ are, of course, singletons, hence the inductive process allows us to insert one direction per step. We may begin the inductive process with any box spline $\phi_{1}$ whose (integer) direction set is a basis for $\mathbb{R}^{d}$ (that box spline is then, up to a normalization constant, the support function of some parallelepiped). As the initial wavelet masks $T_{1}$, we take the tensor product construction (thus, we obtain, up to a linear transformation, the multivariate Haar wavelets). We select any sequence $\Xi=\left(\xi_{1}, \ldots, \xi_{m}\right)$, and, after $m$ insertions obtain the final box spline

$$
\widehat{\phi}(\omega)=\widehat{\phi}_{1}(\omega) \prod_{j=1}^{m} \frac{1-e^{-i 2 \xi_{j} \cdot \omega}}{2 i \xi_{j} \cdot \omega} .
$$

There are $2^{d}-1+m$ boxlets in this construction. The first $2^{d}-1$ ones correspond to the initial tensor product masks (which, we stress, are applied to the smooth $\phi$ ). The other masks, which we index by $\Xi$, have a "triangular structure":

$$
\tau_{\xi_{j}}(\omega):=\tau_{1}(\omega) \frac{1-e^{-i 2 \xi_{j} \cdot \omega}}{2} \prod_{\ell=1}^{j-1} \frac{1+e^{-i 2 \xi_{\ell} \cdot \omega}}{2},
$$

with $\tau_{1}$ the refinement mask of the initial $\phi_{1}$.

Assuming that $m$ is relatively small, most of the boxlets have small support. For example, if we wish to construct $C^{2}$ boxlets, then, independently of the spatial dimension $d$, we may do with $m=3$. In this case, there are $2^{d}-1$ wavelets with "small" support, one with "large" support, and two wavelets with intermediate size of supports.

We remark that, in the above construction, we may start with any refinable (box spline) $\phi_{1}$, provided that a derivation of a tight frame from $\phi_{1}$ is available.

Example. We show that the construction of $\S 3$ is a special case of the construction of this section. Here, $\phi_{1}$ is a four-direction box spline (as defined in (2.1) with multiplicities $\left(0, m_{2}, 0, m_{2}\right)$, and $\phi_{2}$ is a four-direction box spline with multiplicities $\left(m_{1}, 0, m_{1}, 0\right)$ (so that each box spline has only two active directions). The function $\phi_{2}$ is refinable with respect to the dilation matrix $s$ employed in $\S 3$, and with mask (up to an exponential factor) $\cos ^{m_{1}}\left(\omega_{1} / 2\right)$. Since this mask is univariate, one can use our univariate construction from [RS2] to obtain the $\mathrm{T}_{2}$ masks

$$
\mathrm{T}_{2}:=\left\{y_{j}^{\left[m_{1}\right]}\left(\omega_{1}\right): j=1, \ldots, m_{1}\right\} .
$$

As to $\phi_{1}$, the box spline $\phi_{1}$ is refinable with mask (up to an exponential factor) $\cos ^{m_{2}}\left(\omega_{2} / 2\right)$, and hence the same univariate construction can be repeated (as indeed we did). Alternatively, we may assume by induction that $\mathrm{T}_{1}$, the wavelet masks associated with $\phi_{1}$, are already given.

Note that the above discussion shows that the construction of $\S 3$ could have been made gradual: starting with four-direction box spline with multiplicities $\left(0, m_{2}, 0, m_{2}\right)$, we may have appended the four-direction box spline with multiplicities $\left(m_{1}, 0, m_{1}, 0\right)$ step by step: The number of wavelets will be then unchanged, $\left(m_{1}+m_{2}\right)$, but a certain saving in the size of the larger support wavelets can be achieved in this way. 

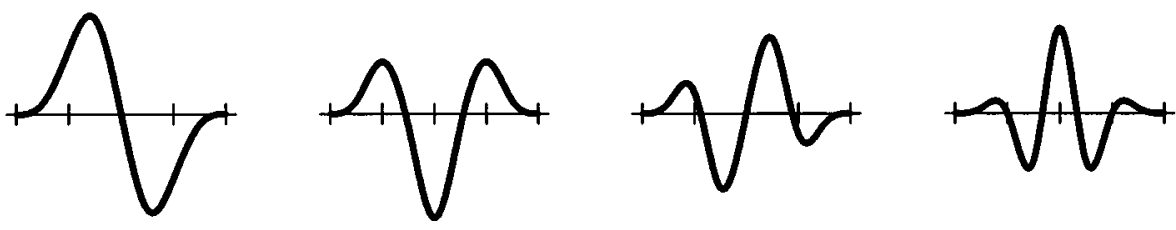

Figure 4.1. The four $C^{2}$ piecewise-cubic wavelets that were constructed in [RS2]. All of them are supported in $[-2,2]$.

Univariate $C^{2}$ cubic splines. In the final example of this section, we employ the general algorithm of this section in the construction of a univariate compactly supported tight frame generated by $C^{2}$ cubic splines. Prior to doing that, we recall that $[\mathrm{RS} 2]$ already provides such a construction. Its construction yields four generators $\left(\psi_{j}\right)_{j=1}^{4}$, each supported in the interval $[-2,2]$ whose Fourier transforms satisfy

$$
\widehat{\psi}_{j}(2 \omega)=\sqrt{\left(\begin{array}{l}
4 \\
j
\end{array}\right)} \frac{\cos ^{4-j}(\omega / 2) \sin ^{4+j}(\omega / 2)}{(\omega / 2)^{4}} .
$$

The graphs of these four functions are given in Figure 4.1.

Here, we construct another $C^{2}$ piecewise-cubic tight frame, generated by 3 wavelets, albeit with larger support. In this construction, we choose $\phi_{1}$ as the constant B-spline, and $\phi_{2}$ as the quadratic B-spline. Thus:

$$
\widehat{\phi}_{1}(\omega)=\frac{1-e^{-i \omega}}{i \omega}, \quad \widehat{\phi}_{2}=\left(\widehat{\phi}_{1}\right)^{3},
$$

and the corresponding masks are

$$
\tau_{1}(\omega)=\frac{1+e^{-i \omega}}{2}, \quad \tau_{2}=\tau_{1}^{3} .
$$

We now choose $T_{1}$ to be the singleton

$$
\mathrm{T}_{1}:=\left\{\frac{1-e^{-i \omega}}{2 i}\right\}
$$

(that corresponds to the Haar wavelet), and choose $\mathrm{T}_{2}$ to consist of two masks:

$$
\mathrm{T}_{2}:=\left\{\left(\frac{1-e^{-i \omega}}{2 i}\right)^{3}, \frac{\sqrt{3}\left(1-e^{-2 i \omega}\right)}{4 i}\right\} .
$$

It is obvious that $T_{1}$ satisfies (b) of (4.1). It is less obvious, but still can be checked directly, that $\mathrm{T}_{2}$ satisfies (c) of (4.1). Thus, by our algorithm, the following three mother wavelets generate a tight frame for $L_{2}(\mathbb{R})$ :

$$
\begin{gathered}
\widehat{\psi}_{1}(\omega)=\frac{1-e^{-i \omega / 2}}{2 i} \widehat{\phi}(\omega / 2), \\
\widehat{\psi}_{2}(\omega)=\frac{1+e^{-i \omega / 2}}{2} \frac{\sqrt{3}\left(1-e^{-2 i \omega}\right)}{4 i} \widehat{\phi}(\omega / 2),
\end{gathered}
$$

and

$$
\widehat{\psi}_{3}(\omega)=\frac{1+e^{-i \omega / 2}}{2}\left(\frac{1-e^{-i \omega}}{2 i}\right)^{3} \widehat{\phi}(\omega / 2),
$$




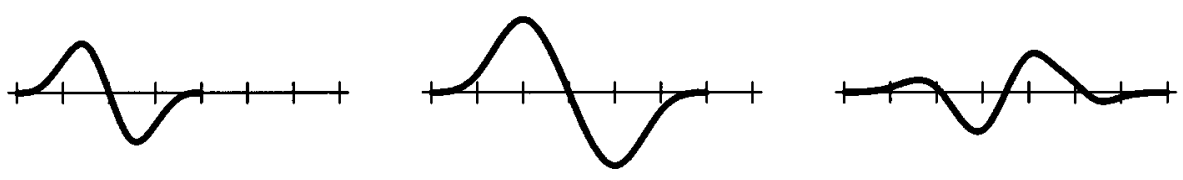

Figure 4.2. The three $C^{2}$ piecewise-cubic wavelets that were constructed here.

where

$$
\widehat{\phi}(\omega)=\left(\frac{1-e^{-i \omega}}{i \omega}\right)\left(\frac{1-e^{-i 2 \omega}}{i 2 \omega}\right)^{3} .
$$

The supports of these wavelets are $[0,4],[0,6],[0,7]$, respectively, and their graphs are shown in Figure 4.2. We note that $\psi_{2}$ and $\psi_{3}$ are splines with integer knots; i.e., in standard wavelet terminology, they belong to $V_{0}$.

\section{ACKNOWLEDGMENTS}

The figures in this article were produced by Matlab. We are indebted to Carl de Boor and Sherm Riemenschneider who, each, made available to us some of their own Matlab m-files.

\section{REFERENCES}

[BHR] C. de Boor, K. Höllig and S.D. Riemenschneider, Box splines, Springer Verlag, New York, (1993). MR 94k:65004

[CD] A. Cohen and I. Daubechies, Non-separable bidimensional wavelets bases, Rev. Mat. Iberoamericana, Vol 9 (1993), 51-137. MR 94k:42047

[CS] C.K. Chui and X. Shi, Inequalities on matrix-dilated Littlewood-Paley energy functions and oversampled affine operators, SIAM J. Math. Anal. 28 (1997), 213-232. CMP 97:06

[CJS] C.K. Chui, K. Jetter, and J. Stöckler, Wavelets and frames on the four-directional mesh, in Wavelets: Theory, Algorithms, and Applications, C. K. Chui, L. Montefusco, L. Puccio (eds.), Academic Press, San Diego, 1994, 213-230. MR 96b:41017

[D1] I. Daubechies, The wavelet transform, time-frequency localization and signal analysis, IEEE Trans. Inform. Theory 36 (1990), 961-1005. MR 91e:42038

[D2] I. Daubechies, Ten lectures on wavelets, CBMS-NSF conference series in applied mathematics, Vol 61, SIAM, Philadelphia, 1992. MR 93e: 42045

[D3] I. Daubechies, Orthonormal bases of compactly supported wavelets, Comm. Pure and Appl. Math. 41 (1988), 909-996. MR 90m:42039

[HW] C. Heil and D. Walnut, Continuous and discrete wavelet transforms, SIAM Review 31 (1989), 628-666. MR 91c:42032

[RS1] A. Ron and Z. Shen, Frames and stable bases for shift-invariant subspaces of $L_{2}\left(\mathbb{R}^{d}\right)$, Canad. J. Math. 47 (1995), 1051-1094. Ftp site: anonymous@ftp.cs.wisc.edu MR 96k: 42049

[RS2] A. Ron and Z. Shen, Affine systems in $L_{2}\left(\mathbb{R}^{d}\right)$ : the analysis of the analysis operator, J. Func. Anal., to appear. Ftp site: anonymous@ftp.cs.wisc.edu

Computer Science Department, University of Wisconsin-Madison, 1210 West Dayton Street, Madison, Wisconsin 53706

E-mail address: amos@cs.wisc.edu

Department of Mathematics, National University of Singapore, 10 Kent Ridge CresCENT, Singapore 119260

E-mail address: matzuows@leonis.nus.sg 